University of Wollongong

Research Online

Faculty of Engineering and Information

Faculty of Engineering and Information

Sciences - Papers: Part A

Sciences

$1-1-2015$

Energy exchange between electric vehicle load and wind generating utilities

\author{
Ahmad Tavakoli \\ University of Tasmania \\ Michael Negnevitsky \\ University of Tasmania, michael.negnevitsky@utas.edu.au \\ Duc Thanh Nguyen \\ TasNetworks Pty. Ltd., dtn156@uow.edu.au \\ Kashem M. Muttaqi \\ University of Wollongong, kashem@uow.edu.au
}

Follow this and additional works at: https://ro.uow.edu.au/eispapers

Part of the Engineering Commons, and the Science and Technology Studies Commons

Research Online is the open access institutional repository for the University of Wollongong. For further information contact the UOW Library: research-pubs@uow.edu.au 


\title{
Energy exchange between electric vehicle load and wind generating utilities
}

\begin{abstract}
This paper proposes a novel approach to energy exchange between electric vehicle (EV) load and wind generation utilities participating in the day-ahead energy, balancing, and regulation markets. An optimal bidding/offering strategy model is developed to mitigate wind energy and EV imbalance threats, and optimize EV charging profiles. A new strategy model is based on optimizing decision making of a wind generating company (WGenCO) in selecting the best option among the use of the balancing or regulation services, the use of the energy storage system (ESS) and the use of all of them to compensate wind power deviation. Energy imbalance is discussed using conventional systems, ESS, and EV-Wind coordination; results are compared and analyzed. Stochastic intra-hour optimization is solved by mixedinteger linear programming (MILP). Uncertainties associated with wind forecasting, energy price, and behavior of EV owners based on their driving patterns, are considered in the proposed stochastic method and validated through several case studies.
\end{abstract}

\section{Keywords}

exchange, energy, between, electric, vehicle, load, wind, generating, utilities

Disciplines

Engineering | Science and Technology Studies

\section{Publication Details}

A. Tavakoli, M. Negnevitsky, D. Nguyen \& K. M. Muttaqi 2015, "Energy exchange between electric vehicle load and wind generating utilities," IEEE Transactions on Power Systems, 31 (2) pp. 1248-1258. 


\title{
Energy Exchange between Electric Vehicle Load and Wind Generating Utilities
}

\author{
A. Tavakoli, Student Member, IEEE, M. Negnevitsky, Senior Member, IEEE, \\ D. T. Nguyen, Member, IEEE, K. Muttaqi, Senior Member, IEEE
}

\begin{abstract}
This paper proposes a novel approach to energy exchange between electric vehicle (EV) load and wind generation utilities participating in the day-ahead energy, balancing, and regulation markets. An optimal bidding/offering strategy model is developed to mitigate wind energy and EV imbalance threats, and optimize EV charging profiles. A new strategy model is based on optimizing decision making of a wind generating company (WGenCO) in selecting the best option among the use of the balancing or regulation services, the use of the energy storage system (ESS) and the use of all of them to compensate wind power deviation. Energy imbalance is discussed using conventional systems, ESS, and EV-Wind coordination; results are compared and analyzed. Stochastic intra-hour optimization is solved by mixed-integer linear programming (MILP). Uncertainties associated with wind forecasting, energy price, and behavior of $\mathrm{EV}$ owners based on their driving patterns, are considered in the proposed stochastic method and validated through several case studies.
\end{abstract}

Index Terms- Electricity market, energy exchange, electric vehicles, energy storage, stochastic optimization.

\section{NOMENCLATURE}

Indices:

$b$

EV

$i$

$m$

$s$

$t$

$w$

Battery storage bank

Electric vehicle fleet

Intrahour (sub-hour) time intervals

A segment of curves

Scenarios

Hourly time intervals

Wind energy units

Parameters:
$D_{E V, s, t, i}$
$E_{E V, t}^{\max }, E_{E V, t}^{\min }$
$N_{E V}$
$N_{E V, s, t, i}$
$N_{I}$
$N_{s}$
$N_{T}$
$N_{W}$

Energy consumption by EV while driving.

Max/Min available energy in EV aggregator. Number of EV fleet.

Plugged-in EV number scenarios in each fleet

Number of intrahour intervals.

Number of scenarios.

Number of hour intervals.

Number of wind energy units.

A. Tavakoli, and M. Negnevitsky are with University of Tasmania, School of Engineering, Hobart 7001, Tasmania, Australia (e-mail: Ahmadt@utas.edu.au, Michael.Negnevitsky@utas.edu.au).

D. T. Nguyen is with TasNetworks Pty. Ltd., Hobart 7004, Tasmania, Australia (e-mail: thanh.nguyen@tasnetworks.com.au).

K. M. Muttaqi is with the University of Wollongong, NSW 2522, Australia. (e-mail: kashem@uow.edu.au).

$P_{b, \text { Max }}^{c h}, P_{b, \text { Max }}^{d c}$
$P_{E V, c h}^{\max }$
$P_{s, t, i}^{w, f}$
$P_{t}^{w, f}$
$S O C_{E V}^{\max , \min }$
$\mathrm{T}$
$\rho_{t}^{D A}$
$\rho_{s, t, i}^{R T}$
$\rho_{t}^{R u p}$
$\rho_{t}^{\text {Rdown }}$
$\rho^{P}$
$\rho^{T}$
$\pi_{s}$

$\varphi_{m}$

$\Delta t$

$i 0, N I$

Variables:

$a_{s, t, k}^{\Delta}, b_{s, t, k}^{\Delta} \quad$ Auxiliary binary variables.

$C_{b, s, t, i}^{d c}$

$E_{E V, s, t, i}$

$I_{b, s, t, i}^{c h}$

$I_{b, s, t, i}^{d c}$

$I_{E V, s, t, i}^{U P}$

$I_{E V, s, t, i}^{D O W N}$

$P_{b, s, t, i}^{c h}$

$P_{E V, s, t, i}^{c h}$

$P_{b, s, t, i}^{d c}$

$P_{b, m, s, t, i}^{d c}$

$P_{s, t, i}^{i m}$

$P_{|i m|, s, t, i}^{i m}$

$P_{t}^{w}, P_{s, t, i}^{w}$

$P o P_{E V, t}^{D A}$ adjusted.

Penalty price ( $\$ / M W h)$.

Probability of scenarios. /discharge curve. time interval. aggregator (MW). (MW). segment $m$ (MW). balancing market (MW). market
Max charge/discharge power of the battery. Max charge power of the EV aggregator. Intra-hour forecasted wind power (MW). Day-ahead forecasted wind power (MW). Maximum/Minimum state of the charge. Time at which specified state of charge is

Day-ahead energy price (\$/MWh).

Real time energy price ( $\$ / M W h)$.

Day-ahead regulation up price (\$/MWh).

Day-ahead regulation down price ( $\$ / \mathrm{MWh})$.

Fixed EV charging tariff ( $\$ / \mathrm{MWh})$.

Slope of segment in linearized charge

Duration of each intrahour time interval.

The beginning and the end of each intrahour

Degradation cost of the discharging battery.

Real-time energy in EV battery.

Charging indicator of the battery storage.

Discharging indicator of the battery storage.

Regulation up indicator of the EVs.

Regulation down indicator of the EVs.

Charge power of the battery storage (MW).

Real-time power drawn by the EV

Discharge power of the battery storage

Discharge power of the battery storage at

Energy imbalance adjusted (provided) by the

Absolute imbalance power of balancing

Day-ahead / Real time wind power (MW).

Preferred operating point (day-ahead powerdrawn) of the EVs (MW). 


$$
\begin{aligned}
& R_{E V, s, t, i}^{D O W N} \\
& R_{E V, s, t, i}^{U P} \\
& \Delta P_{|\Delta| s, t, i}^{W} \\
& \Delta P_{s, t, i}^{w}
\end{aligned}
$$

Regulation down power of EVs (MW). Regulation up power of EVs (MW).

Absolute wind power deviation between Real time and day-ahead scheduling (MW). Wind power deviation between Real time and day-ahead scheduling (MW).

\section{INTRODUCTION}

Electric power generators, transportation systems, and residential houses contribute $41 \%, 23 \%$, and $6 \%$ of the world greenhouse gases, respectively [1]. The strong dependence on foreign energy sources known as "oil addiction", the growing awareness of global warming impacts of $\mathrm{CO}_{2}$ emissions, and high energy efficiency are the driving forces for the increase in the penetration of renewable energy and electric vehicles (EVs) [2, 3].

However, global investment in renewables fell by $14 \%$ during 2013. For example, wind capacity addition reduced to $31 \mathrm{GW}$ in 2013 from $44 \mathrm{GW}$ in 2012. The main cause for this was policy uncertainty in renewable energy [3].

Both wind generation uncertainty, and energy price fluctuations are contributing factors to the decrease of the competitiveness of wind generating companies (WGenCOs) in the energy market [4]. In addition, the current market structure does not usually allow WGenCOs to serve loads directly and there is no coordination between them and distribution companies serving customer loads [5].

An electricity market (e.g., PJM) may have a twosettlement system consisting of two markets - a day-ahead market (DAM) and a real-time balancing market. Generators are paid for any generation that exceeds their day-ahead scheduled quantities and are penalized for generation deviations below their scheduled quantities [6]. Whenever the scheduled day-ahead wind power generation deviates from the real-time market (RTM), the profitability of WGenCOs decreases due to imbalance energy charges for the wind units $[6,7]$.

To mitigate potential wind energy imbalance charges for WGenCOs, the authors in [7], suggested a coordinated scheduling of wind energy units and stora The uncertainties associated with wind forecast ge units. However, the study was based on pumped storage power systems which represent only around $2.2 \%$ of the total generation with efficiency at about $75 \%$; they have high installation costs, and are limited to specific locations $[8,9]$. Although stored energy increases the economic value of wind energy [10], the use of large scale battery-based energy storage systems (ESSs) is currently still prohibitively expensive.

Another factor that contributes to energy imbalance is the increasing penetration of EVs. In the US, it is predicted that by 2020 a quarter and by 2040 two thirds of light-duty vehicles will become EVs [1].

Therefore, power systems are likely to face increasing energy imbalance in both generation and load in the near future. Turning a load on/off or increasing/decreasing the demand can be effective to balance power in the grid. Demand dispatch (DD) is, in some sense, similar to demand response (DR) with the main difference that DR is used only to shed loads at peak times, while DD is intended to be used actively at all times [11]. The coordinated control of millions of controllable loads (including EVs) can potentially balance power in the grid.

The EVs, when aggregated in a sizeable number, can play an important role in regulation service due to much faster ramping capability than gas turbines through vehicle-to-grid (V2G) technology [9-11]. The EV participation in the energy and ancillary service markets has been investigated in several studies. In [12], the EV participation considered bidirectional V2G interactions. Though V2G can be both unidirectional and bidirectional, the unidirectional V2G is expected to be implemented first as it requires less infrastructure and reduces the battery degradation by not requiring additional cycling for bidirectional power flow [10]. In [10, 12], unidirectional smart charging with EV participation in the energy and regulation markets is studied without considering the stochastic nature of the process.

Currently EV usage is in its initial stage; when EV penetration becomes strong and influences the grid parameters, control of a large number of EVs to balance the entire power system will represent a challenge for power utilities. Several studies have demonstrated the benefits of coordination between wind power generators and EVs in power networks. The authors of [13] have examined the effect of EV integration in a wind-thermal power system on emissions produced. In [14], a stochastic unit commitment model is used to simulate wind-thermal power system scheduling with different charging patterns for EVs to reduce operating costs of a power system. However, these studies did not consider the intra-hour variability of the EV charging behavior and wind energy generation which limits potential benefits of energy dispatch in the power system. In [15], coordinated wind-EV in three energy dispatching approaches, i.e., valley searching, interruptible and variable rate dispatching, is used without considering economic issues. Study [5] proposed coordinating unidirectional vehicle-togrid (V2G) services with energy trading. In [5], EV aggregators did not participate in the regulation market. In $[16,17]$, it has been shown that the highest benefits for EV owners are expected through participation in regulation markets.

The imbalanced energy exchange based on a dedicated coordination between EV load aggregators and WGenCOs can potentially increase the competitiveness of WGenCOs and EV-load customers in the energy market. This paper develops an optimal bidding/offering strategy for EV load demands in coordination with a WGenCO, thereby maximizing the WGenCO's competitiveness, optimizing EV charging profiles and mitigating imbalance energy provided by the balancing market. The EV Aggregator participates in the energy and ancillary service markets while the WGenCO participates in the day-ahead energy and balancing markets.

The main contributions of the paper are as follows:

- The development of a two-stage stochastic linear programming (SLP)-based optimal offering/bidding strategy model for the coordinated EV-Wind units 
participating in the day-ahead energy, balancing, and regulation markets.

- The development of a SLP-based optimal offering strategy model for the ES-Wind units participating in the day-ahead energy, and balancing markets.

- A new strategy model based on optimal decision making for selecting between the balancing, regulation services, and/or using ESS for a WGenCO to compensate wind power deviation.

- Comprehensive comparisons of three different cases comprising conventional systems (WGenCO without energy storage), WGenCO with ESS, and a coordinated EV-Wind energy exchange for dealing with energy imbalance.

- Considering the uncertainties associated with wind forecast, energy price, and EV owners' behavior based on driving patterns.

The rest of this paper is organized as follows. Section II discusses the market framework. Section III provides a mathematical model formulation. Scenario generation and reduction are presented in Section IV. A case study is described in Section V. Section VI concludes the paper.

\section{MARKET FRAMEWORK}

The day-ahead market and a real-time balancing market are the two settlement systems considered in this paper. Participants in the DAM submit supply-offers/demand-bids to the system operator. These participants also submit supplyoffers for the regulation capacity, and they may later submit revised regulation quantities, which are different from dayahead offer quantities, without any penalty imposed [17]. The time framework for the DAM is the whole day $d$, which is cleared at 10am of the day $(d-1)$. The balancing market ensures the real-time balance between generation and demand by offsetting the difference between the real-time operation and the last energy program cleared in the markets. For this reason, this market remains open until 10 minutes before the delivery hour [18].

Wind generation and EV load aggregators participate as price takers in the DAM by hourly offering/bidding amounts that are based on the day-ahead forecast while energy and price variations occur within minutes (i.e. intra-hour) [19].

In this paper, three different strategies are considered to deal with the energy imbalance for a WGenCO participating in short-term electricity markets (DAM and balancing). In the subsections below, these strategies are demonstrated using conventional systems (WGenCO without storage), WGenCO with the energy storage system, and a power system with a coordinated EV-Wind energy exchange.

\section{A. Conventional Systems}

WGenCOs participate in the DAM and balancing market. The imbalance charge is imposed on the WGenCO to balance energy in the power system due to deviation of the RTM [4, $6,7]$. The WGenCO's payoff in this method is as follows:

$$
\rho_{t}^{D A} P_{t}^{w}-\rho^{P}\left|P_{t}^{w}-P_{s, t, i}^{w}\right|-\rho_{s, t, k}^{R T}\left(P_{t}^{w}-P_{s, t, i}^{w}\right)
$$

According to (2), the wind energy deviation between dayahead and real-time is considered as the energy imbalance:

$$
\Delta P_{s, t, i}^{w}=P_{t}^{w}-P_{s, t, i}^{w}=P_{s, t, i}^{i m}
$$

\section{B. WGenCO with the Energy Storage System}

It is assumed that battery storage belongs to the WGenCO participating in the DAM and balancing market. The authors in [4] and [7] proposed a scheduling strategy for the coordination of wind and storage units without any flexibility for the WGenCO to adapt when the storage units fail.

Our paper proposes a new scheduling strategy, which considers optimal decision making for WGenCOs in selecting between the balancing market and ESS to compensate for wind power deviations. The WGenCO can decide whether to use the ESS or not based on penalties, energy prices, maintenance requirements and other factors. According to (3), the wind energy deviation between the DAM and RTM can be compensated by the battery storage system and balancing market. The optimization determines the one which is the most efficient.

$\Delta P_{s, t, i}^{w}=P_{t}^{w}-P_{s, t, i}^{w}=\left(P_{b, s, t, i}^{d c}-P_{b, s, t, i}^{c h}\right)+P_{s, t, i}^{i m}$

A degradation cost from the battery bank charging/discharging is considered in this method. The WGenCO's payoff is as follows:

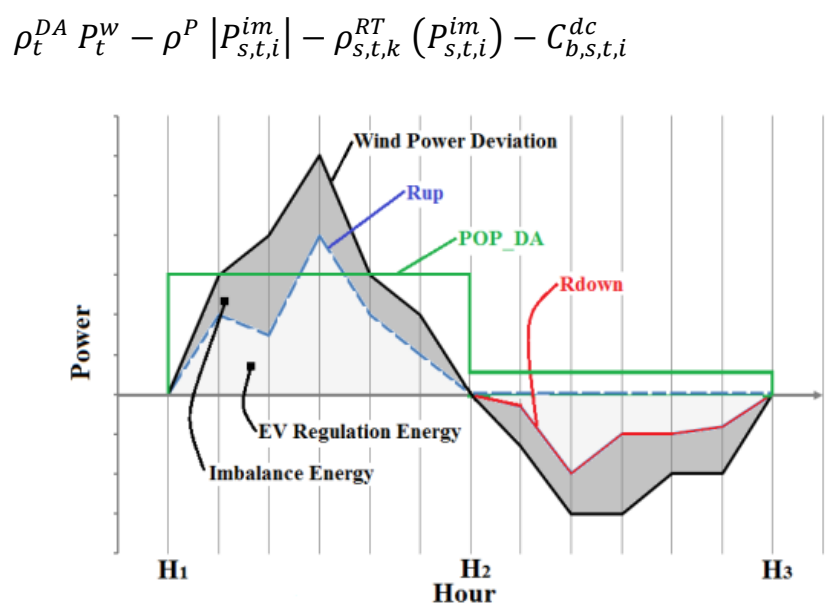

Fig. 1 Coordination between EV demand and wind power deviation in energy and regulation market.

\section{Coordinated EV-Wind Energy Exchange}

In the method discussed above, the WGenCO participates in the short-term electricity market. The EV aggregator participates as dispatchable load in the energy and ancillary service markets by submitting energy bids and regulation offers. The amount of regulation contracted is the total amount by which power can deviate from a baseline level. The baseline is often called the preferred operating point, or POP [11]. The term POP itself comes from ancillary services markets. It represents the average level of operation for a market participant providing regulation services [10]. It is assumed that the EV aggregator can deviate from the dayahead power-drawn (or POP) to amend wind imbalance energy by reducing or increasing their charging rate with 
consideration of EV aggregator energy constraints. Therefore, the offer price in the day-ahead market does not change in the real-time market while offer quantities can be revised [17]. The penalties are not imposed for revising the day-ahead power drawn offer quantities [16]. When real-time wind energy exceeds the forecasted day-ahead wind energy, the EV aggregator regulates down with more charging, and vice versa (see Fig.1).

The wind energy deviation between DAM and RTM is compensated by structured regulation, which is provided by the EV aggregator, and unstructured regulation, which is provided by the balancing market as follows:

$\Delta P_{s, t, i}^{w}=P_{t}^{w}-P_{s, t, i}^{w}=\left(R_{E V, s, t, i}^{U P}-R_{E V, s, t, i}^{D O W N}\right)+P_{s, t, i}^{i m}$

Therefore, the WGenCO can select between regulation and balancing markets based on penalties, energy prices, lack of EVs, and other factors. The WGenCO's payoff in coordination with EV aggregators in this part is:

$\rho_{t}^{D A} P_{t}^{w}-\rho^{P}\left|P_{s, t, i}^{i m}\right|-\rho_{s, t, k}^{R T} \Delta P_{s, t, i}^{w}$

$-\left(\rho_{t}^{\text {Rup }} R_{E V, s, t, i}^{U P}+\rho_{t}^{\text {Rdown }} R_{E V, s, t, i}^{D O W N}\right)$

Real-time power drawn by the EV aggregator is given by:

$P_{s, E V, t, i}^{c h}=P o P_{E V, t}^{D A}-R_{E V, s, t, i}^{U P}+R_{E V, s, t, i}^{D O W N}$

Hence, the EV aggregator's revenue is obtained by selling ancillary services, as well as selling energy to its clients at a fixed price $\left(\rho^{T}\right)$. In this paper, the tariff charged to EV clients is assumed to be constant (fixed). The aggregator encourages $\mathrm{EV}$ owners to join in by offering an attractive price for charging in comparison with petrol and energy prices.

The EV aggregator's cost is associated with buying energy for EV charging. Hence, the EV aggregator's payoff is represented as:

$-\left(\rho_{t}^{D A}-\rho^{T}\right) P o P_{E V, t}^{D A}+\left[\rho_{s, t, i}^{R T}-\rho^{T}\right]\left(P o P_{E V, t}^{D A}-P_{s, E V, t, i}^{c h}\right)$

$+\left(\rho_{t}^{R u p} R_{E V, s, t, i}^{U P}+\rho_{t}^{\text {Rdown }} R_{E V, s, t, i}^{D O W N}\right)$

\section{MATHEMATICAL MODEL FORMULATION}

In this section, the problems of optimal dispatch for three different WGenCO scheduling strategies are formulated and presented. These problems are solved as a two-stage mixedinteger stochastic program [20-21]. The first-stage variable is decided before stochastic variables with the hourly day-ahead input parameters such as $\rho_{t}^{D A}, \rho_{t}^{\text {Rup }}$, and $\rho_{t}^{\text {Rdown }}$. The secondstage variable is dependent on scenarios with sub-hourly (intra-hourly) RT input parameters such as $\rho_{s, t, i}^{R T}, N_{E V, s, t, i}$, and $P_{s, t, i}^{w, f}$.

\section{A. Conventional Systems}

The objective function (9) is to maximize the revenue from selling the day-ahead wind energy minus the cost of energy imbalance [4, 6, 7]. The intra-hour based wind power deviation between real-time and day-ahead schedules is in (10), and generation limits are given in (11)-(12). Equations (13)-(16) are a linear representation of the absolute value of variable $\Delta P_{s, t, i}^{w}$ for the mixed-integer linear programming (MILP) formulation [4]. In equation (15), $\mathrm{M}$ is the upper bound of $\left(\Delta P_{|\Delta| s, t, i}^{w}-\Delta P_{s, t, i}^{w}\right)$, and in (16), $\mathrm{M}$ is the upper bound of $\left(\Delta P_{|\Delta| s, t, i}^{w}+\Delta P_{s, t, i}^{w}\right)$. These equations are proved in Appendix.

$$
\begin{aligned}
& \mid \operatorname{Max} \sum_{s=1}^{N_{s}} \pi_{s}\left[\sum_{t=1}^{N_{T}} \sum_{w=1}^{N_{w}} \rho_{t}^{D A} P_{t}^{w}\right] \\
& -\pi_{s} \cdot\left[\frac{1}{N_{I}} \sum_{i=1}^{N_{I}} \sum_{t=1}^{N_{T}} \sum_{w=1}^{N_{w}} \rho_{s, t, i}^{R T} \Delta P_{s, t, i}^{w}\right] \\
& -\pi_{s} \cdot\left[\frac{1}{N_{I}} \sum_{i=1}^{N_{I}} \sum_{t=1}^{N_{T}} \sum_{w=1}^{N_{w}} \rho^{P} \Delta P_{|\Delta| s, t, i}^{w}\right] \\
& \Delta P_{s, t, i}^{w}=P_{t}^{w}-P_{s, t, i}^{w}=P_{s, t, i}^{i m} \\
& P_{s, t, i}^{w} \leq P_{s, t, i}^{w, f} \\
& P_{t}^{w} \leq P_{t}^{w, f} \\
& \Delta P_{|\Delta| s, t, i}^{w}-\Delta P_{s, t, i}^{w} \geq 0 \\
& \Delta P_{|\Delta| s, t, i}^{w}+\Delta P_{s, t, i}^{w} \geq 0 \\
& \Delta P_{|\Delta| s, t, i}^{w}-\Delta P_{s, t, i}^{w} \leq M a_{s, t, i}^{\Delta} \\
& \Delta P_{|\Delta| s, t, i}^{w}+\Delta P_{s, t, i}^{w} \leq M\left[1-a_{s, t, i}^{\Delta}\right]
\end{aligned}
$$

\section{B. WGenCO with the Energy Storage System}

The objective function (17) is to maximize the revenue from selling the day-ahead wind energy minus the cost of energy imbalance and battery charging/discharging costs [6]. The intra-hour-based wind power deviation between RT and day-ahead schedule is given in (18). The wind power deviation has two terms including imbalance, and battery charging/discharging energy. Equations (19)-(22) are a linear form of the absolute value of variable $P_{s, t, i}^{i m}$ for MILP formulation, where $\mathrm{M}$ is a large positive number [4]. Battery charging/discharging and imbalance power constraints are in (23)-(26). Constraints presented in (11)(16) are used as well.

The charging/discharging cost depends directly on the depth of discharge (DoD) and the number of cycles to failure of the battery [16]. As the depth of aggregated battery discharge increases, the number of cycles to failure decreases. The piecewise linear representation of the concave discharge cost curve of EV batteries in the proposed MIP formulation is shown in (27) and (28).

$$
\begin{aligned}
& \mid \operatorname{Max} \sum_{s=1}^{N_{S}} \pi_{s} \cdot\left[\sum_{t=1}^{N_{T}} \sum_{w=1}^{N_{w}} \rho_{t}^{D A} P_{t}^{w}\right] \\
& -\pi_{s} \cdot\left[\frac{1}{N_{I}} \sum_{i=1}^{N_{I}} \sum_{t=1}^{N_{T}} \sum_{w=1}^{N_{w}} \rho_{s, t, i}^{R T} \cdot P_{s, t, i}^{i m}\right] \\
& -\pi_{s} \cdot\left[\frac{1}{N_{I}} \sum_{i=1}^{N_{I}} \sum_{t=1}^{N_{T}} \sum_{w=1}^{N_{w}} \rho^{P} P_{|i m|, s, t, i}^{i m}\right]
\end{aligned}
$$


$\left.-\pi_{s} \cdot\left[\frac{1}{N_{I}} \sum_{i=1}^{N_{I}} \sum_{t=1}^{N_{T}} C_{b, s, t, i}^{d c}\right]\right)$

$\Delta P_{s, t, i}^{w}=P_{s, t, i}^{i m}+P_{b, s, t, i}^{d c}-P_{b, s, t, i}^{c h}$

$P_{|i m|, s, t, i}^{i m}-P_{s, t, i}^{i m} \geq 0$

$P_{|i m|, s, t, i}^{i m}+P_{s, t, i}^{i m} \geq 0$

$P_{|i m|, s, t, i}^{i m}-P_{s, t, i}^{i m} \leq M b_{s, t, i}^{\Delta}$

$P_{|i m|, s, t, i}^{i m}+P_{s, t, i}^{i m} \leq M\left[1-b_{s, t, k}^{\Delta}\right]$

$P_{|i m|, s, t, i}^{i m} \leq \Delta P_{|\Delta| s, t, i}^{w}$

$0 \leq P_{b, s, t, i}^{d c} \leq P_{b, \operatorname{Max}}^{d c} I_{b, s, t, i}^{d c}$

$0 \leq P_{b, s, t, i}^{c h} \leq P_{b, \text { Max }}^{c h} I_{b, s, t, i}^{c h}$

$I_{s, b, t, i}^{d c}+I_{s, b, t, i}^{c h} \leq 1$

$C_{b, s, t, i}^{d c}=P_{m}^{\min } \cdot I_{b, s, t, i}^{d c}+\sum_{m=1}^{N_{M}} \varphi_{m} \cdot P_{b, m, s, t, i}^{d c}$

$$
\sum_{m=1}^{N_{M}} P_{b, m, s, t, i}^{d c}+P_{m}^{\min } I_{b, s, t, i}^{d c}=P_{E V, s, t, i}^{d c}
$$

\section{Coordinated EV-Wind Energy Exchange}

The objective function (29) is to maximize the profits of the EV aggregator and the WGenCO according to (6) and (8). In this strategy, the wind power deviation between DAM and RTM is compensated by regulating the down/up charging power of the EV aggregator $\left(R_{E V, s, t, i}^{D O W N} / R_{E V, s, t, i}^{U P}\right)$ and by the energy imbalance $\left(P_{s, t, i}^{i m}\right)$ provided by the grid, as given (30).

The energy balance equation for the EV aggregator is given in (31). The EV energy capacity in each intrahour $\left(E_{E V, s, t, i}\right)$ is the EV energy capacity in prior intrahour $\left(E_{E V, s, t, i-1}\right)$ plus energy charged by drawing power from the grid $\left(P_{E V, s, t, i}^{c h} . \Delta t\right)$ minus energy consumed by EVs while driving. The regulation capacity of the EV aggregator increases when the numbers of charging EVs increase and, vice versa as given in (31).

Constraints presented in (11)-(16) and (19)-(23) are also used here. Constraints for the EV's POP, capacity to increase the charging rate for regulation down $\left(R_{E V, s, t, i}^{D O W N}\right)$, capacity to decrease the charging rate for regulation up $\left(R_{E V, s, t, i}^{U P}\right)$ are given in (32)-(36). In (37), the status of regulation down or up is determined. The EV energy constraint is presented in (38). The constraint (39) imposes limits at the beginning and at the end of each interval of the energy capacity of the EV aggregator. The constraint (40) specifies the level of SOC to be reached by time $(\mathrm{T})$ for a specified $\mathrm{EV}$ client. This constraint is an option for clients to set up the desirable SOC for their EVs at the time of expected commuting (T). For example, the EV client wants to have the battery fully charge (i.e., $100 \%$ SOC) by the departure time (for example, 5:00pm) to go back home.

\section{SCENARIO GENERATION AND REDUCTION TECHNIQUES}

There are several different scenario generation and reduction techniques for stochastic programming [22]. The
Monte Carlo simulations are applied to generate scenarios in [22]. In [23], the time series models are used to generate scenarios for prices in electricity markets. The most common scenario-reduction technique is based on Kantorovich distance [24]. In [25], a scenario generation and reduction technique for price forecasting is based on the roulette wheel mechanism.

$$
\begin{aligned}
& \left\lfloor\operatorname{Max} \sum_{s=1}^{N_{s}} \pi_{s} \cdot\left[\sum_{t=1}^{N_{T}} \sum_{w=1}^{N_{w}} \rho_{t}^{D A} P_{t}^{w}\right]\right. \\
& -\pi_{s} \cdot\left[\sum_{t=1}^{N_{T}} \sum_{E V=1}^{N_{E V}}\left(\rho_{t}^{D A}-\rho^{T}\right) P o P_{E V, t}^{D A}\right] \\
& +\pi_{s} \cdot\left[\frac{1}{N_{I}} \sum_{t=1}^{N_{T}} \sum_{E V=1}^{N_{E V}} \sum_{i=1}^{N_{I}}\left[\rho_{s, t, i}^{R T}-\rho^{T}\right]\left(R_{E V, s, t, i}^{U P}-R_{E V, s, t, i}^{D O W N}\right)\right] \\
& -\pi_{s} \cdot\left[\frac{1}{N_{I}} \sum_{i=1}^{N_{I}} \sum_{t=1}^{N_{T}} \sum_{w=1}^{N_{w}} \rho_{s, t, i}^{R T} \cdot \Delta P_{s, t, i}^{w}\right] \\
& \left.-\pi_{s} \cdot\left[\frac{1}{N_{I}} \sum_{i=1}^{N_{I}} \sum_{t=1}^{N_{T}} \sum_{w=1}^{N_{w}} \rho^{P} \cdot P_{|i m|, s, t, i}^{i m}\right]\right) \\
& \Delta P_{s, t, i}^{w}=P_{s, t, i}^{i m}+R_{E V, s, t, i}^{U P}-R_{E V, s, t, i}^{D O W N} \\
& E_{E V, s, t, i}=E_{E V, s, t, i-1}+P_{E V, s, t, i}^{c h} \cdot \Delta t \\
& -\left(1-N_{E V, s, t, i}\right) D_{E V, s, t, i} \\
& P o P_{E V, t}^{D A} \leq P_{E V, c h}^{\max } \cdot N_{E V, s, t, i} \\
& P o P_{E V, t}^{D A}+R_{E V, s, t, i}^{D O W N} \leq P_{E V, c h}^{\max } \cdot N_{E V, s, t, i} \\
& R_{E V, s, t, i}^{U P} \leq P o P_{E V, t}^{D A} \\
& R_{E V, s, t, i}^{D O W N} \leq P_{E V, c h}^{\max } \cdot N_{E V, s, t, i} \cdot I_{E V, s, t, i}^{D O W N} \\
& R_{E V, s, t, i}^{U P} \leq P_{E V, c h}^{\max } \cdot N_{E V, s, t, i} \cdot I_{E V, s, t, i}^{U P} \\
& I_{E V, s, t, i}^{D O W N}+I_{E V, s, t, i}^{U P} \leq 1 \\
& E_{E V, t}^{\min } \cdot N_{E V, s, t, i} \cdot S O C_{E V}^{\min } \leq E_{E V, s, t, i} \\
& \leq E_{E V, t}^{\max } \cdot N_{E V, s, t, i} S O C_{E V}^{\max } \\
& E_{E V, s, t, i 0}=E_{E V, s, t-1, N I} \\
& E_{E V, s, t, T}=E_{E V, t}^{\max } N_{E V, s, t, i} S O C_{E V}^{\max }
\end{aligned}
$$

In this paper, scenario generation and reduction techniques are used for simulating wind speed, energy price, and the number of EVs engaged as follows.

\section{A. Wind and Energy Price Scenarios}

Wind speed forecasting for the next day can be obtained from numerical meteorological programs, however, forecasts are never perfect. The Auto Regressive Moving Average (ARMA) model is used to simulate wind speed forecast errors [26-28]. The ARMA (p, q) model for a stochastic process $\mathrm{X}$ is defined as:

$\mathrm{x}(\mathrm{t})=\sum_{j=1}^{p} \alpha(j) \cdot x(t-j)+z(t)+\sum_{j=1}^{q} \beta(j) \cdot z(t-j)$

where $\mathrm{p}$ is autoregressive parameters $\alpha_{1}, \alpha_{2}, \ldots, \alpha_{\mathrm{p}}$, and $\mathrm{q}$ is moving average parameters $\beta_{1}, \beta_{2}, \ldots, \beta_{\mathrm{q}} ; \mathrm{Z}(\mathrm{t})$ is a random Gaussian variable with standard deviation $\sigma$ [28]. 
The estimation and adjustment of ARMA models have been investigated in literature. In this paper, the first order of the ARMA model, ARMA $(1,1)$, is used to simulate wind speed forecasting errors. This approach has been suggested in [26], [27]:

$\Delta \mathrm{V}(t)=\alpha \Delta \mathrm{V}(t-1)+\mathrm{Z}(\mathrm{t})+\beta \mathrm{Z}(t-1)$

where $\Delta \mathrm{V}(\mathrm{t})$ is the wind speed forecast error at the time $(t)$ forecast; and $\alpha$, and $\beta$ are parameters.

The estimation of parameters $\alpha$ and $\beta$ for a given wind speed forecast is done as suggested in [26]. ARMA parameters are obtained by minimizing the difference in the root mean square error (RMSE) between the simulated ARMA model and the wind speed measurement data [7], [27].

The real wind speed $V(t)$ is calculated as the sum of the wind speed forecast $V^{f}(t)$ and the wind speed forecast error:

$\mathrm{V}(\mathrm{t})=\mathrm{V}^{\mathrm{f}}(t)+\Delta \mathrm{V}(t)$

Once a large number of scenarios are generated, the wind speed scenarios are transformed into power scenarios through the power conversion curve for each wind turbine [4], [18].

Similarly, Auto Regressive Integrated Moving Average (ARIMA) models have been applied to forecast electricity prices, which appear non-stationarily when the processes present a periodic or seasonal pattern [28], [29].

\section{B. EV Penetration Scenarios}

Any driving profile has a commute time including morning and evening with the start and end times, and a commute distance. Major commuting would normally begin between $7 \mathrm{am}$ and $9 \mathrm{am}$ to go to work and between $5 \mathrm{pm}$ and $8 \mathrm{pm}$ to come back from work. For all other times, the EVs are assumed to be available to be plugged into the electricity grid [30].

The EV availability at each interval has associated unplanned departure and arrival probabilities. The number of EVs is considered to be random, and Monte Carlo simulations are used to generate possible scenarios.

The total number of EVs is 1p.u. It is assumed that on average, from 2a.m. to 5a.m., 98\% of EVs are plugged-in with a standard deviation of $5 \%$. For commute periods, on average, $20 \%$ of EVs are plugged-in with a standard deviation $10 \%$, and during other periods it is assumed that $85 \%$ of EVs are plugged-in with a standard deviation of $20 \%$ [12]. The availability of EVs in various time periods is shown in Table I.

TABLE I THE AVAILABILITY OF EVs IN VARIOUS TIME INTERVALS

\begin{tabular}{ccccccc}
\hline Hour & $2: 00$ & $5: 00$ & $\mathbf{7 : 0 0}$ & $9: 00$ & $\mathbf{1 7 : 0 0}$ & $20: 00$ \\
& $5: 00$ & $7: 00$ & $\mathbf{9 : 0 0}$ & $17: 00$ & $\mathbf{2 0 : 0 0}$ & $2: 00$ \\
\hline \hline St.dev (\%) & 5 & 20 & 10 & 20 & 10 & 20 \\
\hline Mean (pu) & 0.98 & 0.85 & 0.20 & 0.85 & 0.20 & 0.85 \\
\hline
\end{tabular}

\section{Scenario Reduction}

In stochastic optimization problems with various inherent uncertainties, a large number of scenarios can emerge. It can, therefore, be computationally expensive. Therefore, a technique for reducing the number of scenarios is required.

In this paper, the scenario reduction algorithm is based on [30], [31]. The basic idea of the scenario reduction is to eliminate scenarios with low-probabilities, and cluster similar scenarios [7], [33]. The new probability of a preserved scenario is determined as the sum of its initial probability and the probabilities of similar scenarios that have been eliminated. We used SCENRED as a tool for scenario reduction [33]. SCENRED contains three reduction algorithms: the fast backward method, a mix of fast backward/forward methods and a mix of fast backward/backward methods. The algorithms have different computational performance, and the choice of algorithms for a certain problem depends on the size of the problem and the required solution accuracy. The strategy used in [31-33] recommends that the optimal deletion of scenarios should be determined by a conceptual algorithm called backward reduction. If the number of preserved scenarios is small (strong reduction) the optimal selection of a single scenario may be repeated recursively until a prescribed number of preserved scenarios is selected. This strategy provides the basic concept of the conceptual algorithm called forward selection. In this paper, the fast backward/forward method is selected to reduce the number of scenarios [32].

\section{CASE STUDIES}

To test the proposed model, a WGenCO with a single wind farm is assumed to participate in a day-ahead energy market. The capacity of the wind farm is $200 \mathrm{MW}$, which is a relatively small farm compared to the wholesale energy market. The WGenCO is a price-taker; it is not a dominant player in the wholesale energy market. 10,000 scenarios are reduced to just ten using scenario reduction techniques presented in [33]. Fig. 2 shows the intrahourly wind power generation forecasted for these ten scenarios. Fig. 3 shows the day-ahead energy price, regulation up/down prices, and intra-hour real time energy price scenarios. Fig. 4 shows the intra-hourly $\mathrm{EV}$ penetration forecasted for the same ten scenarios. We consider the worst conditions to occur when peak demand and high regulation prices coincide with the lowest penetration of EVs (see Fig. 3-4). The number of intrahour intervals is 6 (10 min each).

The maximum EV charging power is assumed to be $7.3 \mathrm{~kW}$, and the energy capacity of each EV is $27.4 \mathrm{kWh}$. Average annual driving distance of an EV is assumed $20,000 \mathrm{~km}$ with an average daily distance of $52.91 \mathrm{~km}$. The required energy for an EV is $9 \mathrm{kWh} /$ day with an average of $5.87 \mathrm{~km} / \mathrm{kWh}$ [16], [18], [21]. In this paper, the fixed charging tariff is assumed to be $\$ 0.01 / \mathrm{kWh}$ [12]. We assume that the required energy for driving in one direction is the same as that of returning to the starting point. For the EV aggregator, we consider two EV penetration scenarios 1,000 and 10,000 EVs. The cycle efficiency is $83.6 \%$ for a charging/discharging efficiency of $95 \%[16,18]$. The EV fleet has its own commute time based on the region, city, traffic patterns, etc. In this paper, the number of EV fleets is assumed to be one with commute intervals between $7 \mathrm{a} . \mathrm{m}$. and 9a.m., and between 5p.m. and 8p.m. However, the 
equations provided in the paper are general and can be used for any number of EV fleets. In this paper, $100 \%$ SOC is considered for departure times to represent the worst case scenario.

The capacity of a battery bank of the ESS is assumed similar to the capacity of $10,000 \mathrm{EVs}$. The current price of a complete battery pack is $\$ 600 / \mathrm{kWh}$.

Four cases are considered for the investigation of two important issues: the payoff, and generation and demand dispatch. The four cases are defined as follows:

- Case A) Conventional systems: The WGenCO without ESS participates in the energy market.

- Case B) ES-wind: The WGenCO with ESS participates in the energy market.

- Case C) $1 K-E V$-Wind: The WGenCO in coordination with 1,000 EVs participates in the energy and regulation markets.

- Case D) 10K-EV-Wind: The WGenCO in coordination with $10,000 \mathrm{EVs}$ participates in the energy and regulation markets.

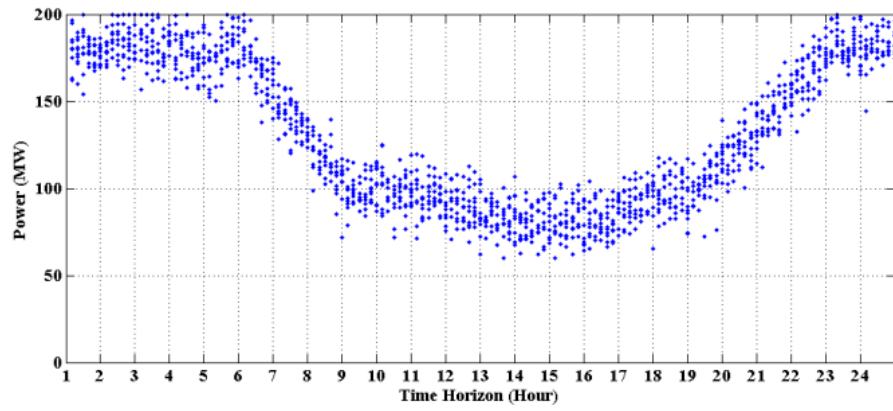

Fig. 2 The intra-hourly wind power generation forecasted for ten scenarios.

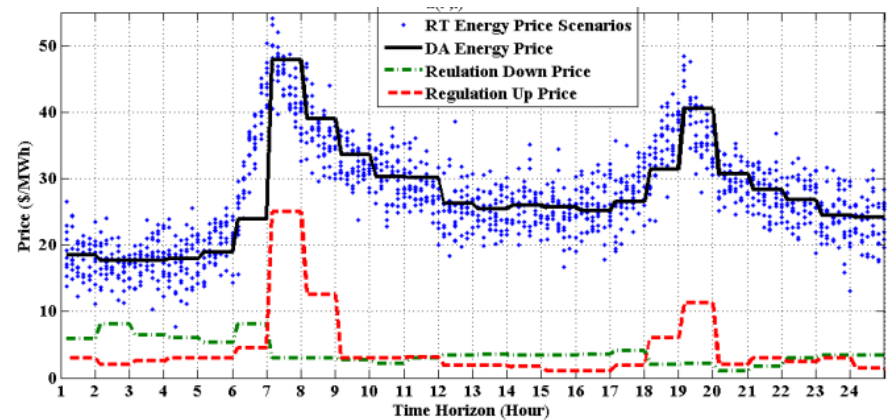

Fig. 3 The day-ahead energy price, regulation up/down prices, and intra-hour real time energy price scenarios.

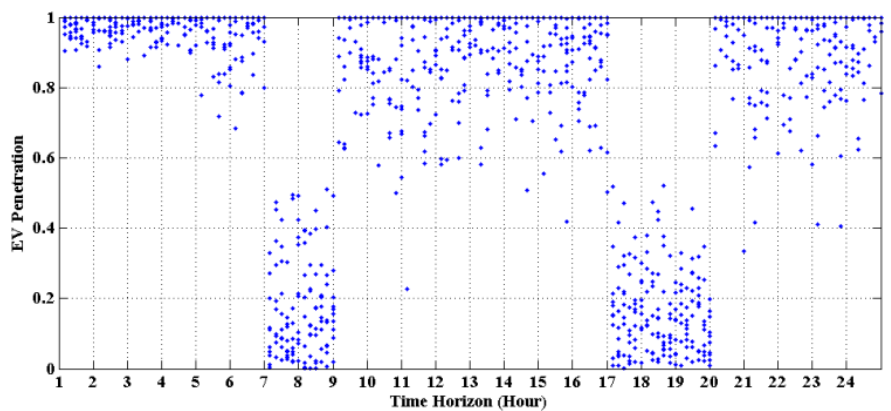

Fig. 4 The intra-hourly EV penetration forecasted for ten scenarios.

\section{A. Payoff Analysis}

Table II shows the total WGenCO's payoffs for all cases when the penalty price is $\$ 30 / \mathrm{MWh}$. The total payoff for Case D is $\$ 79,888.98$, while the expected payoffs in cases A and $\mathrm{B}$ are $\$ 77,023.94$ and $\$ 77,064.63$, respectively. The difference between the two payoffs in case D and case A is $\$ 2,869.04(3.72 \%)$, while the difference between the two payoffs in Case B and Case A is just $\$ 40.69$ (0.05\%). It is clear that using the battery storage at a penalty price of $\$ 30 / \mathrm{MWh}$ is not affordable. The EV penetration impact on the payoffs is obvious when comparing cases $\mathrm{C}$ and $\mathrm{D}$. Table II shows that the payoff in Case D exceeds that of Case $\mathrm{C}$ by $1.86 \%$.

Tables III-V and Figs 5-7 show the impact of the penalty price on the WGenCO's payoffs for different cases. It is clear that with the increasing penalty price, the day-ahead energy sale revenue and the total payoff decrease with more conservative day-ahead generation offers. However, with the EV-wind coordination, the total payoff with respect to the penalty price remains almost constant (see Fig. 5). For example, while penalty price changes from $\$ 10 / \mathrm{MWh}$ to $\$ 150 / \mathrm{MWh}$, the total payoff in cases D, C, B, and A decreases by $2.21 \%, 7.42 \%, 8.02 \%$ and $10.01 \%$, respectively. It is clear that the total payoff under variable penalty price is more sustainable (stable) with the 10K-EV-Wind coordination (see Fig. 5).

Comparing results presented in Tables III-V shows that the difference between the two payoffs in Case D and Case A at the penalty price of $\$ 10 / \mathrm{MWh}$ is $\$ 384(0.4 \%)$, while this difference is $\$ 6683(8.4 \%)$ at the penalty price of $\$ 150 / \mathrm{MWh}$. Therefore, the effectiveness of coordinating EVs with wind generation becomes more apparent for penalty prices greater than \$20/MWh (see Fig. 5).

Comparing Case B with cases $\mathrm{C}$ and $\mathrm{A}$ shows that the total payoff in Case B is greater than in Case A for penalty prices greater than $\$ 40 / \mathrm{MWh}$. The total payoff in Case B is lower than in Case $\mathrm{C}$ although they get closer with higher penalty prices (see Fig. 5), since the battery discharging cost is more affordable under higher penalty prices.

Comparing cases $\mathrm{D}$ and $\mathrm{C}$ shows that the appropriate capacity of the EV aggregator is an important factor in coordinating the EVs and WGenCOs. Benefits of the use of $\mathrm{EV}$ aggregators of sufficient capacity include higher total payoffs, lower imbalance charges and less conservative dayahead generation offers. However, even a smaller number of EVs offer better results in comparison with cases A and B.

Fig. 6 shows imbalance charges provided by the balancing market versus penalty prices. It is clear that the imbalance charge in Case D is less than in the other cases. Fig. 7 demonstrates that an increase in penalty price decreases the $\mathrm{EV}$ regulation cost for the $\mathrm{WGenCO}$ (or the $\mathrm{EV}$ regulation profitability for the EV aggregator) when wind deviations decrease, thus the EV regulation contribution is lower. The battery charging/discharging cost for Case B would increase with the increase in the penalty price, because imbalance charges imposed by the balancing market are more expensive than the battery charging/discharging cost under higher penalty prices; thus the ESS contribution is higher. 
TABLE II

WGenCO's PAYOFFS IN DIFFERENT CASES AT THE \$30 PENALTY PRICE

\begin{tabular}{ccccc}
\hline Cases & Case A & Case B & Case C & Case D \\
\hline $\begin{array}{c}\text { DA Energy Sale } \\
\text { Revenue (\$) }\end{array}$ & 81132.56 & 81177.86 & 83395.12 & 99763.90 \\
\hline $\begin{array}{c}\text { DA Revenue } \\
\text { Adjustment (\$) }\end{array}$ & -875.06 & -702.45 & -2307.46 & -17520 \\
\hline Imbalance Charge (\$) & -3233.56 & -3071.77 & -2491.57 & -589.20 \\
\hline Regulation Cost (\$) & - & - & -195.60 & -1620.61 \\
\hline Discharging Cost (\$) & - & -339.01 & - & - \\
\hline Payoff $\mathbf{( \$ )}$ & 77023.94 & 77064.63 & 78400.48 & 79888.98 \\
\hline
\end{tabular}

TABLE III

IMPACT OF THE PENALTY PRICE ON WGenCO'S PAYOFFS IN CASE A

\begin{tabular}{|c|c|c|c|c|}
\hline Penalty Price (\$/MWh) & 10 & 50 & 100 & 150 \\
\hline $\begin{array}{c}\text { DA Energy Sale } \\
\text { Revenue (\$) }\end{array}$ & 78966.02 & 79304.49 & 76935.06 & 75953.17 \\
\hline $\begin{array}{c}\text { DA Revenue } \\
\text { Adjustment (\$) }\end{array}$ & 4853.88 & -1375.28 & -720.16 & -545.94 \\
\hline Imbalance Charge (\$) & -3136.93 & -2332.88 & -2411.29 & -2745.66 \\
\hline Payoff (\$) & 80682.97 & 75596.32 & 73803.61 & 72661.56 \\
\hline
\end{tabular}

TABLE IV

IMPACT OF THE PENALTY PRICE ON WGenCO'S PAYOFFS IN CASE B

\begin{tabular}{ccccc}
\hline Penalty Price (\$/MWh) & $\mathbf{1 0}$ & $\mathbf{5 0}$ & $\mathbf{1 0 0}$ & $\mathbf{1 5 0}$ \\
\hline $\begin{array}{c}\text { DA Energy Sale } \\
\text { Revenue (\$) }\end{array}$ & 78721.16 & 79605.04 & 79097.86 & 77505.53 \\
\hline $\begin{array}{c}\text { DA Revenue } \\
\text { Adjustment (\$) }\end{array}$ & 4046.73 & -1018.64 & -272.77 & -149.45 \\
\hline Imbalance Charge (\$) & -2330.48 & -1763.76 & -939.59 & -920.54 \\
\hline Discharging Cost (\$) & -1.49 & -987.91 & -3272.19 & -2254.82 \\
\hline Payoff (\$) & 80435.92 & 75834.72 & 75907.64 & 74329.83 \\
\hline
\end{tabular}

TABLE V

IMPACT OF THE PENALTY PRICE ON WGenCO'S PAYOFFS IN CASE D

\begin{tabular}{ccccc}
\hline Penalty Price (\$/MWh) & $\mathbf{1 0}$ & $\mathbf{5 0}$ & $\mathbf{1 0 0}$ & $\mathbf{1 5 0}$ \\
\hline $\begin{array}{c}\text { DA Energy Sale } \\
\text { Revenue (\$) }\end{array}$ & 103290.28 & 98285.83 & 96980.28 & 96154.44 \\
\hline $\begin{array}{c}\text { DA Revenue } \\
\text { Adjustment (\$) }\end{array}$ & -21730 & -16370 & -15380 & -14760 \\
\hline Imbalance Charge (\$) & -492.93 & -492.99 & -486.48 & -560.03 \\
\hline Regulation Cost (\$) & -2339.87 & -1650.21 & -1543.62 & -1489.86 \\
\hline Payoff (\$) & 81067.35 & 79775.13 & 79568.18 & 79344.94 \\
\hline
\end{tabular}

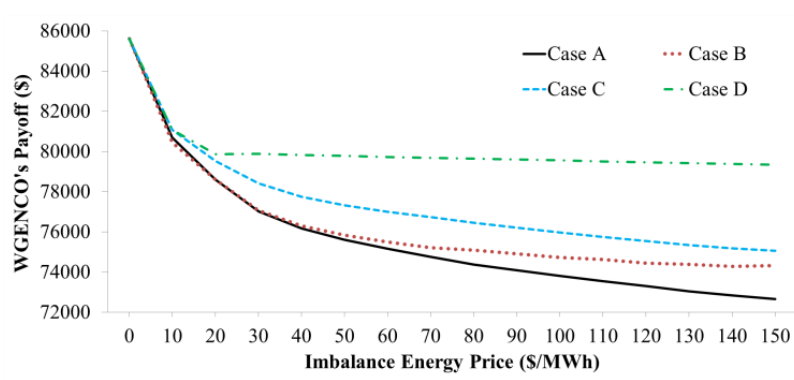

Fig. 5 The WGenCO's payoff versus penalty prices.

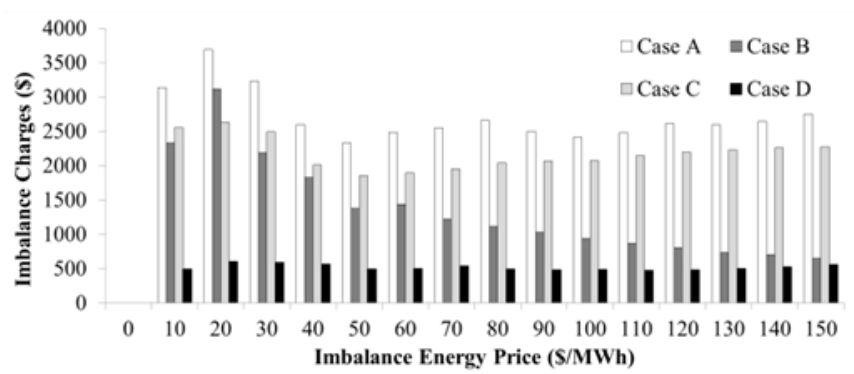

Fig. 6 Imbalance charges provided by balancing market versus penalty prices

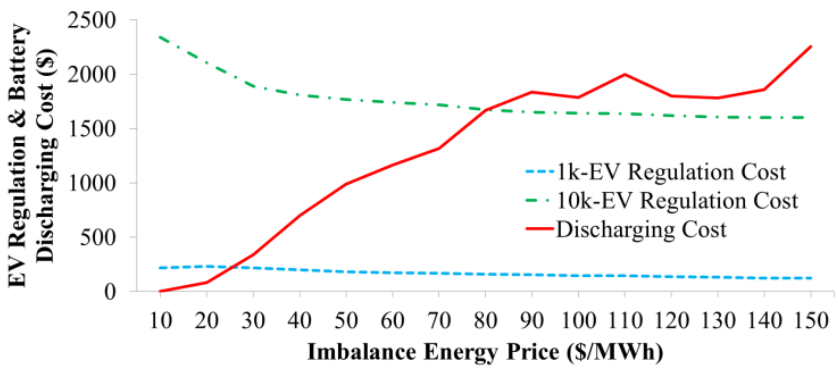

Fig. 7 EV regulation and battery discharging cost versus penalty prices.

\section{B. Demand and Generation Dispatch analysis}

Wind power generation and EV load demand dispatch in Case D at penalty prices of $\$ 10 / \mathrm{MWh}$ and $\$ 150 / \mathrm{MWh}$ are shown in Figs 8 and 9, respectively. These figures show dayahead wind power (Pw-DA), real-time wind power (Pw-RT), wind power deviation $(\Delta P w)$, day-ahead EV charging schedule (POP-DA), EV regulation up/down, and energy imbalance provided by the balancing market $\left(P^{i m}\right)$. Figs 10 and 11 show wind power generation and battery bank dispatch in Case $B$ at penalty prices of $\$ 10 \mathrm{MWh}$ and $\$ 150 / \mathrm{MWh}$, respectively.

It can be seen that the Pw-RT schedules in Figs 8, 9, and 10 look very similar, but the schedule in Fig. 11 differs noticeably. This demonstrates that the penalty price increase has a greater impact in Case B than in Case D. For instance, in Table VI, the total Pw-RT per day in cases B and D are 2,977 MWh, and 3,111 MWh, respectively, at the penalty price of $\$ 150 / \mathrm{MWh}$. However, the total Pw-RT per day in cases $\mathrm{B}$ and $\mathrm{D}$ are the same for the $\$ 10 / \mathrm{MWh}$ penalty price. This demonstrates that the effectiveness of the coordinated EV-wind energy exchange becomes more apparent when penalty prices are higher. From Table VI, it can also be observed that the total Pw-RT in Case D remains almost unchanged irrespective of the penalty price.

If we now compare the total $\Delta P W$ per day for all cases under the $\$ 10 / \mathrm{MWh}$ penalty price, we find that this parameter is much higher in Case D (this can be attributed to the less conservative day-ahead generation offers). We can also find that the total $P^{i m}$ per day under any penalty price is smaller in Case D than in all other cases (this fact is particularly apparent under the $\$ 10 / \mathrm{MWh}$ penalty price).

Results presented in Figs 8, 9 and Table VI also demonstrate that the increase in the penalty price decreases the total up/down EV regulation contributions - the total up/down EV regulation at \$10/MWh and $\$ 150 / \mathrm{MWh}$ penalty prices are 761.9 MWh and 584.1 MWh, respectively.

Results presented in Figs 10, 11 and Table VI show that the increase in penalty price leads to higher battery charging/discharging costs (Case B). The total charging/discharging cost at penalty prices of $\$ 10 / \mathrm{MWh}$ and $\$ 150 / \mathrm{MWh}$ are 4.7 MWh and 69.8 MWh, respectively. Battery discharge power increases under the higher penalty prices, because the battery charging/discharging cost is more affordable than imbalance charges imposed by the balancing market under higher penalty prices. 
TABLE VI

TOTAL REAL-TIME WIND POWER GENERATION, WIND POWER DEVIATION AND ENERGY IMBALANCE PER DAY

\begin{tabular}{|c|c|c|c|c|c|}
\hline $\begin{array}{c}\text { Total } \\
\text { (MWh) } \\
\end{array}$ & $\begin{array}{c}\text { Penalty } \\
\text { Price } \\
\end{array}$ & Case A & Case B & Case C & Case D \\
\hline Pw-RT & \multirow{3}{*}{$\begin{array}{c}10 \\
\text { (\$/MWh) }\end{array}$} & 3138 & 3138 & 3138 & 3137 \\
\hline$\Delta P \boldsymbol{w}$ & & 313.69 & 216.56 & 325.12 & 811.24 \\
\hline$P^{i m}$ & & 313.69 & 211.86 & 255.42 & 49.29 \\
\hline Pw-RT & \multirow{3}{*}{$\begin{array}{c}100 \\
(\$ / M W h)\end{array}$} & 2896 & 2990 & 2976 & 3116 \\
\hline$\Delta P w$ & & 24.11 & 79.16 & 60.58 & 609.2 \\
\hline$P^{i m}$ & & 24.11 & 9.39 & 20.74 & 4.86 \\
\hline Pw-RT & \multirow{3}{*}{$\begin{array}{c}150 \\
(\$ / M W h)\end{array}$} & 2867 & 2977 & 2949 & 3111 \\
\hline$\Delta P \boldsymbol{w}$ & & 18.30 & 73.59 & 46.1 & 588.8 \\
\hline$P^{i m}$ & & 18.30 & 4.317 & 15.167 & 3.733 \\
\hline
\end{tabular}

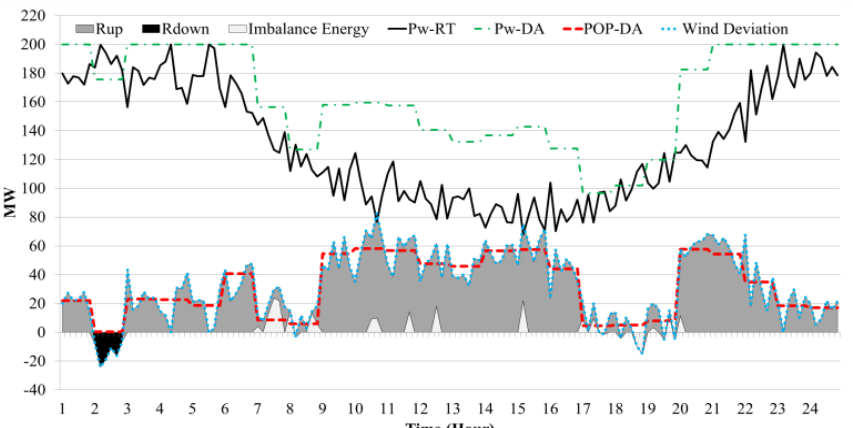

Fig.8 Wind power generation and EV demand schedule in Case D under the $\$ 10 / \mathrm{MWh}$ penalty price.

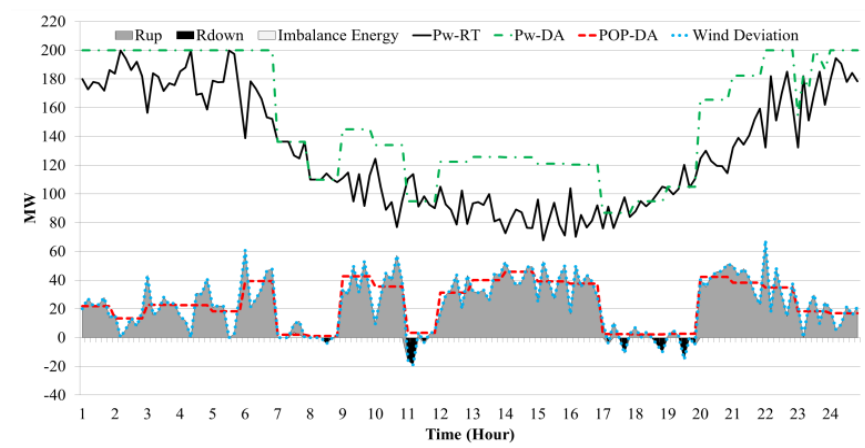

Fig. 9 Wind power generation and EV demand schedule in Case D under the $\$ 150 / \mathrm{MWh}$ penalty price.

\section{CONCLUSION}

Effective coordination between a WGenCO participating in the short-term electricity market and an EV aggregator participating in the energy and ancillary service markets increases the WGenCO's competitiveness and mitigates wind and EV energy imbalance threats. This paper has introduced a stochastic optimal scheduling strategy. The strategy has been demonstrated on conventional systems (WGenCO without storage), WGenCO with ESS, and a power system with a coordinated EV-Wind energy exchange. The proposed strategy has been developed using model based-optimal decision making. It offers flexibility in selecting between the balancing, regulation services, and/or ESS for a WGenCO to compensate for wind power deviations. Comparisons of the coordinated EV-Wind energy exchange with the other two cases reveal that

- the coordinated EV-wind energy exchange ensures that the WGenCO payoff remains constant under changing penalty prices;

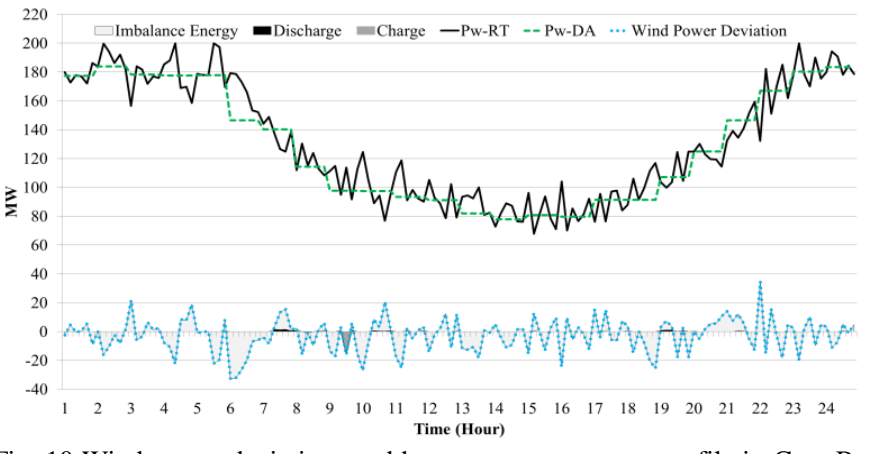

Fig. 10 Wind power deviations and battery energy storage profile in Case B under the $\$ 10 / \mathrm{MWh}$ penalty price.

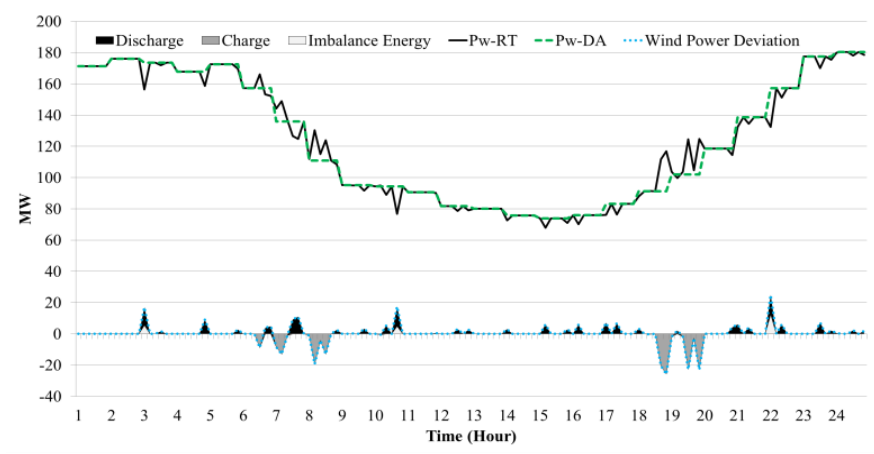

Fig. 11 Wind power generation schedule and battery energy storage profile in Case B under the $\$ 150 / \mathrm{MWh}$ penalty price.

- the effectiveness of the coordinated EV-wind energy exchange becomes more apparent under higher penalty prices;

- benefits offered by EV aggregators of sufficient capacity include higher total payoffs, lower imbalance charges and less conservative day-ahead generation offers (however, even a smaller number of EVs offer better results in comparison with the other two cases);

- the total energy imbalance adjusted by the balancing market decreases extremely under the coordinated EVwind energy exchange.

\section{APPENDIX}

To represent the absolute value of variable $x$ in a linear form for MILP formulation, the following equations are used:

$0 \leq|x|-x \leq M a_{s, t, i}^{\Delta}$

$0 \leq|x|+x \leq M\left[1-a_{s, t, i}^{\Delta}\right]$

$a_{s, t, i}^{\Delta} \in\{0,1\}, M$ is a large positive number

For $x>0$ :

if $a_{s, t, i}^{\Delta}=1 \rightarrow\left\{\begin{array}{c}0 \leq|x|-x \leq M \\ |x|=-x\end{array}\right.$, impossible

if $a_{s, t, i}^{\Delta}=0 \rightarrow\left\{\begin{array}{c}|x|=x \\ 0 \leq|x|+x \leq M\end{array}\right.$, possible

For $x<0$ :

if $a_{s, t, i}^{\Delta}=1 \rightarrow\left\{\begin{array}{c}0 \leq|x|-x \leq M \\ |x|=-x\end{array}\right.$, possible 
if $a_{s, t, i}^{\Delta}=0 \rightarrow\left\{\begin{array}{c}|x|=x \\ 0 \leq|x|+x \leq M\end{array}\right.$, impossible

\section{REFERENCES}

[1] M. H. K. Tushar, C. Assi, M. Maier, and M. F. Uddin, "Smart Microgrids: Optimal Joint Scheduling for Electric Vehicles and Home Appliances", IEEE Trans. Smart Grid, vol.5, no.1, pp.239-250, Jan. 2014.

[2] C. Guille and G. Gross, "Design of a Conceptual Framework for the V2G Implementation," IEEE Energy 2030 Conference, vol., no., pp.1-3, 17-18 Nov. 2008

[3] Frankfurt School-UNEP Centre/BNEF, "Global Trends in Renewable Energy Investment 2014", [Online] Available: http://www.unep.org/pdf/Green_energy_2013-Key_findings.pdf.

[4] L. V. L. Abreu, M. E. Khodayar, M. Shahidehpour, and Lei Wu, "RiskConstrained Coordination of Cascaded Hydro Units With Variable Wind Power Generation," IEEE Trans. Sustain. Energy, vol.3, no.3, pp.359368, Jul. 2012

[5] A. T. Al-Awami, and E. Sortomme, "Coordinating Vehicle-to-Grid Services With Energy Trading", IEEE Trans. Smart Grid, vol.3, no.1, pp.453-462, Mar. 2012

[6] PJM Manual 11: Energy \& Ancillary Services Market Operations, Revision 51, Effective Date: 2012.

[7] M. E. Khodayar, and M. Shahidehpour, "Stochastic Price-Based Coordination of Intrahour Wind Energy and Storage in a Generation Company", IEEE Trans. Sustain. Energy, vol.4, no.3, pp.554-562, Jul. 2013.

[8] Wu Chenye, H. Mohsenian-Rad, Jianwei Huang, "Vehicle-toAggregator Interaction Game", IEEE Trans. Smart Grid, vol.3, no.1, pp.434-442, Mar. 2012

[9] A. Rogers, A. Henderson, X. Wang, and M. Negnevitsky, "Compressed air energy storage: Thermodynamic and economic review," PES General Meeting, 2014 IEEE Conference \& Exposition, vol., no., pp.1, 5, 27-31 Jul. 2014.

[10]E. Sortomme, and M. A. El-Sharkawi, "Optimal Charging Strategies for Unidirectional Vehicle-to-Grid", IEEE Trans. Smart Grid, vol.2, no.1, pp.131-138, Mar. 2011

[11]A. Brooks, E. Lu, D. Reicher, C. Spirakis, and B. Weihl, "Demand Dispatch", IEEE Power and Energy Magazine, vol.8, no.3, pp.20,29, May-Jun. 2010

[12] Sortomme, E.; El-Sharkawi, M.A., "Optimal Scheduling of Vehicle-toGrid Energy and Ancillary Services", IEEE Trans. Smart Grid, vol.3, no.1, pp.351-359, Mar. 2012.

[13]L. Goransson, S. Karlsson, and F. Johnsson, "Integration of plug-in hybrid electric vehicles in a regional wind-thermal power system," $J$. Energy Policy, vol. 38, no. 10, pp. 5482-5492, Oct. 2010

[14]Liu Cong, W. Jianhui A. Botterud, Yan Zhou, and A. Vyas, "Assessment of Impacts of PHEV Charging Patterns on Wind-Thermal Scheduling by Stochastic Unit Commitment", IEEE Trans. Smart Grid, vol.3, no.2, pp.675-683, Jun. 2012.

[15] Wu Ting, Yang Qiang, Bao Zhejing, Yan Wenjun, "Coordinated Energy Dispatching in Microgrid With Wind Power Generation and Plug-in Electric Vehicles", IEEE Trans. Smart Grid, vol.4, no.3, pp.1453-1463, Sep. 2013.

[16]J. Tomic and W. Kempton, "Using fleets of electric-drive vehicles for grid support," J. Power Sources, vol. 168, no. 2, pp. 459-468, Jun. 2007.

[17] S. I. Vagropoulos, and A. G. Bakirtzis, , "Optimal Bidding Strategy for Electric Vehicle Aggregators in Electricity Markets", IEEE Trans. Power Syst., vol.28, no.4, pp.4031-4041, Nov. 2013.

[18] J. M. Morales, A. J. Conejo, and J. Perez-Ruiz, "Short-Term Trading for a Wind Power Producer", IEEE Trans. Power Syst., vol.25, no.1, pp.554-564, Feb. 2010.

[19]M. E. Khodayar, and M. Shahidehpour, "Hourly Coordination of Electric Vehicle Operation and Volatile Wind Power Generation in SCUC", IEEE Trans. Smart Grid, vol.3, no.3, pp.1271-1279, Sep. 2012

[20]S.-E. Fleten and T. K. Kristoffersen. Stochastic programming for optimizing bidding strategies of a Nordic hydropower producer. European Journal of Operational Research, 181(2), 916-928, 2007.

[21]H. Pandzic, J. M. Moralesb, A. J. Conejo, and I. Kuzled, "Offering model for a virtual power plant based on stochastic programming", Applied Energy, vol. 105, PP. 282-292, May 2013.
[22] Li Tao, M. Shahidehpour, and Li Zuyi, "Risk-Constrained Bidding Strategy With Stochastic Unit Commitment," IEEE Trans. Power Syst., vol.22, no.1, pp.449,458, Feb. 2007

[23]M. A. Plazas, A. J. Conejo, and F. Prieto, "Multimarket optimal bidding for a power producer," IEEE Trans. Power Syst., vol. 20, no. 4, pp. 2041-2050, Nov. 2005.

[24]S Mahir. "Modeling the Diversification Benefit of Transmission Investments." Master thesis, Second Level, Univ. KTH, Sweden, 2012

[25]N. Amjady, J. Aghaei, and H. A. Shayanfar, "Stochastic Multiobjective Market Clearing of Joint Energy and Reserves Auctions Ensuring Power System Security", IEEE Trans. Power Syst., vol.24, no.4, pp.1841-1854, Nov. 2009.

[26]L. Soder, "Simulation of wind speed forecast errors for operation planning of multiarea power systems," in Proc. 2004 Int. Conf. Proc. Probabilistic Methods Applied to Power Systems, Sep. 12-16, 2004, pp. 723-728.

[27]A. Boone, "Simulation of Short-Term Wind Speed Forecast Errors Using a Multi-Variate ARMA $(1,1)$ Time-Series Model,” Master thesis, Univ. KTH Royal Institute of Technology, Sweden, 2005.

[28] S. A. Gabriel, A. J. Conejo, J. D. Fuller, B. F. Hobbs, and C. Ruiz, Complementarity Modeling in Energy Markets, ser. International Series in Operations Research \& Management Science. New York, NY, USA: Springer, 2012.

[29] J. Contreras, R. Espinola, F. J. Nogales, A. J. Conejo, "ARIMA models to predict next-day electricity prices," IEEE Trans. Power Syst., vol.18, no.3, pp.1014,1020, Aug. 2003

[30]Electrification Coalition, "Electrification roadmap: Revolutionizing transportation and achieving energy security," Nov. 2013 [Online]. Available:http://www.electrificationcoalition.org/sites/default/files/SAF 1213_EC-Roadmap_v12_Online.pdf.

[31]J. Dupacova, N. Growe-Kuska, and W. Romisch, "Scenario reduction in stochastic programming: an approach using probability metrics," Math. Prog. Series A, vol. 3, pp. 493-511, 2003.

[32]N. Growe-Kuska, H. Heitsch, and W. Romisch, "Scenario reduction and scenario tree construction for power management problems," in Proc. IEEE Power Tech Conf., Bologna, Italy, Jun. 2003, vol. 3, pp. 23-26.

[33] GAMS/SCENRED Documentation [Online]. Available: http://www.gams.com/docs/document.htm

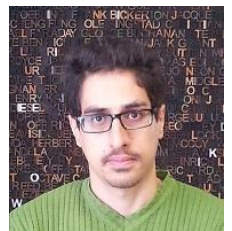

Ahmad Tavakoli (S'09) is currently pursuing the $\mathrm{Ph} . \mathrm{D}$. degree in power engineering and computational intelligence at University of Tasmania, Hobart, Australia. His research interests include power systems protection, operation and control, electricity market analysis, and smart grid.

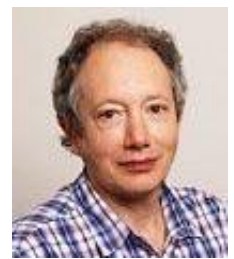

Michael Negnevitsky (M'95-SM'07) received the B.S.E.E. (Hons.) and Ph.D. degrees from the Byelorussian University of Technology, Minsk, Belarus, in 1978 and 1983, respectively. Currently, he is Chair Professor in Power Engineering and Computational Intelligence, University of Tasmania, Australia. He is a Chartered Professional Engineer and Fellow of Engineers Australia.

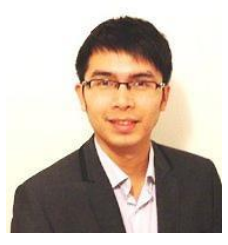

Duy Thanh Nguyen (S'09-M'12) received the B.E. (Hons.) and Ph.D. degrees from the University of Tasmania, Hobart, Australia, in 2009 and 2012, respectively. Currently Dr. Nguyen is working as a Smart Grid Specialist for Aurora Energy Pty. Ltd, Hobart, Australia. His special fields of interests are network planning, operation and control, electricity market analysis, and smart grid.

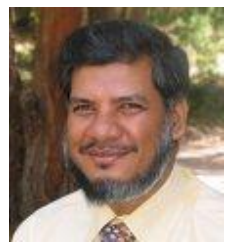

Kashem M. Muttaqi (M'01-SM'05) received the Ph.D. degree from Multimedia University, Malaysia, in 2001. Currently, he is an Associate Professor at the School of Electrical, Computer, and Telecommunications Engineering, University of Wollongong, Wollongong, Australia. $\mathrm{He}$ was associated with the University of Tasmania, Australia as a Research Fellow/Lecturer/Senior Lecturer from 2002 to 2007, and with the Queensland University of Technology, Australia as a Research Fellow from 2000 to 2002. 